\title{
MS39-P02 | A Novel X-RAy Diffraction TeChNique FOR IN-Situ ObSERVATIONS OF Cathode/Anode Reaction in Electrolytes
}

Kubicek, Sabine (KAI GmbH, Villach, AUT)

Surface sensitive in-situ techniques are helpful to get a deeper understanding of electrochemical reactions because they can give information about electrode reaction products during the reaction process. Structural and phase properties of thin solid films are commonly measured by grazing incident X-ray diffraction. Ex-situ measurements are sometimes not feasible because of alteration of deposits in contact with air, moisture or aging within the time gap between formation and measurement. Therefore in-situ X-ray diffraction cells for electrochemical experiments were developed. Such cell designs are limited due to geometry restrictions and, more importantly, due to radiation intensity loss because of the interaction of the beam with thick window materials or the electrolyte. Therefore, existing cells are frequently used in combination with synchrotron radiation.

We present a novel grazing incident X-ray diffraction setup for in-situ observations of cathode/anode reaction in electrolytes, which uses a backside-illuminated working electrode. The cell uses a polyimide foil with a thin sputtered metal layer as working electrode. The X-ray penetrates the foil with the thin metal layer from the backside and interacts with the metal layer and reaction products formed at the interface between working electrode and electrolyte solution. Because of the relatively small intensity losses, lab-scale equipment with a copper radiation source is appropriate. The cell can be operated in a three-electrode setup without any restrictions in current density distribution or electrolyte flow and can be used for any electrochemical method like voltammetry, deposition in galvanostatic and potentiostatic mode and electrochemical impedance spectroscopy (EIS). 